\title{
Energy Management Strategies for Hybrid Electric Vehicles
}

\author{
P. Caratozzolo \\ Cenidet \\ Internado Palmira s/n \\ 62490 Cuernavaca, Morelos \\ Mexico
}

\author{
M. Serra \\ IRI, UPC-Csic \\ Llorens i Artigas 4-6 \\ 08028 Barcelona, Catalonia \\ Spain
}

\author{
J. Riera \\ IRI, UPC-Csic \\ Llorens i Artigas 4-6 \\ 08028 Barcelona, Catalonia \\ Spain
}

\begin{abstract}
Coupled with the accelerated search of "clean generation" of electric energy is the growing need for alternative energy source in urban transportation that can reduce the overall impact on air pollution. Hybrid Electric Vehicles, HEV, are offering the best possibility for the use of new energy sources, because electricity can result from a transformation with high efficiency in systems with electric drives and components.

In this paper the authors pretend to show the possible application of a particular motion system, the Joint System, JS, in a simplified HEV model. A special energy management strategy must be applied in order to take into account the operation modes of the JS and the particular constraints linked to the structure and configuration of an HEV.

An application-oriented overview of a rule-based control is presented and is demonstrated the suitability for the control of HEVs.
\end{abstract}

\section{INTRODUCTION}

Hybrid Electric Vehicles, HEV, are offering the best possibility for the use of new energy sources in urban transportation, reducing the overall impact on air pollution while obtain minimum fuel consumption.

In this paper the authors pretend to show the possible application of a particular motion system, the Joint System, $\mathrm{JS}$, in a simplified HEV model. A special energy management strategy must be applied in order to take into account the operation modes of the JS and the particular constraints linked to the structure and configuration of an HEV.

Efforts are being made to improve the HEV electrical subsystems and specially the system-level integration and optimization. In that sense, this paper pretends to contribute in the improvement of the hybrid drivetrain configuration applying the new motion system developed, the JS.

In the first part of this paper, a generic HEV is described, with series configuration. Second, a detailed explanation of the modes of operation of the JS is presented.

Finally, the central part of this work that is the management of energy flows is presented. As far as this aspect concerns, an application-oriented overview of a rulebased control is presented and is demonstrated the suitability for the control of HEVs.

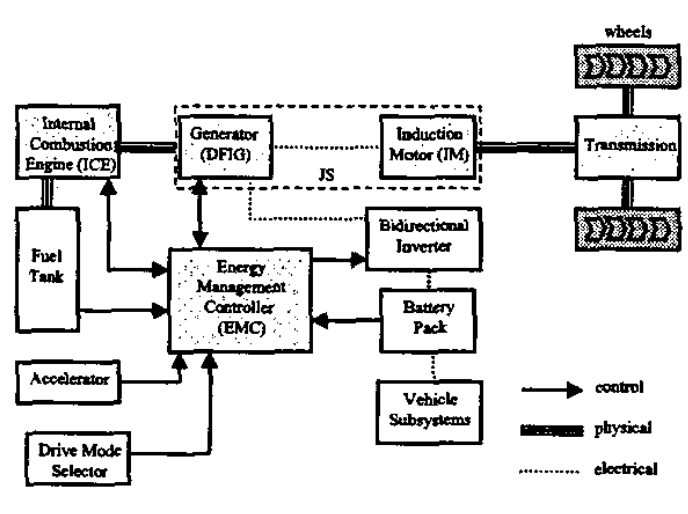

Figure 1: Series HEV System Schematic

\section{HYBRID ELECTRIC VEHICLE}

The terminology Hybrid Vehicle is generally applied to vehicles that use an internal combustion engine, ICE, associated to equipment to generate electric energy "on board". The electric energy is then used to feed a traction electric motor, usually an induction motor, IM, associated to the ICE in order to power the wheels of the vehicle [1].

The HEV combines the particular features of ICE and IM to minimize transient engine operation and to take advantage of the IM suitability to acceleration.

In a typical series design for an HEV, the ICE mechanical output is first convert into electricity using a generator, commonly a permanent magnet synchronous generator, PMSG.

A new proposed electric generation scheme consists in replacing the PMSG by a double-fed induction generator, DFIG, like in Fig. 1. With this scheme the DFIG is used as a variable-voltage variable-frequency (VVVF) generator to supply the IM wheels drive with the appropriated power supply. It is possible to consider jointly the DFIG and the IM, giving rise to what we denominate the Joint System, JS. 


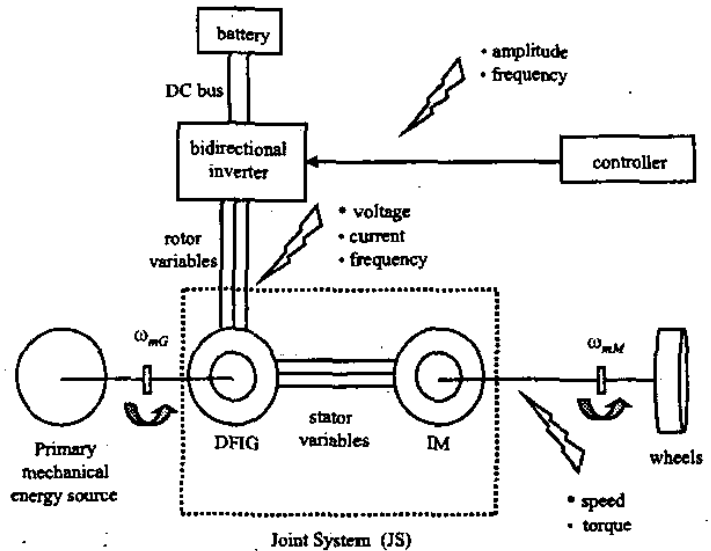

Figure 2. System Configuration

\section{JOINT SYSTEM}

The behavior of the JS can be explained as follows: IS is an autonomous motion system that can drive a rotating load, delivering the demanded output power.

The DFIG is driven through the rotor shaft by a constant speed prime mover, which can be the ICE in the case of an HEV.

With the constant speed of the prime mover it is expected that the performance of the primary energy source can be optimized. In the case of the ICE, optimization can be obtain by reducing the fuel consumption working not at a unique constant speed but at different predetermined fixed speeds (three for example, as explained in section VI, Figure 7).

A bank of batteries is connected to the JS rotor circuit via a bidirectional inverter, delivering a slip power, that is a fraction of total power.

In this system the DFIG is able to operate in two modes delivering leading or lagging stator current to the IM. Depending on the operating condition, power may flow from. the converter to the rotor or in the reverse direction.

As the mechanical power delivered from the prime mover is normally larger than the electrical energy required from the converter at the rotor side, the control system has to be rated only for slip power, which is only a fraction of stator power.

The resulting reduction in the inverter costs makes this slip recovery system very attractive in all applications where energy saving is an important factor [2].

JS has been modeled by a system of complex ordinary differential equations of sixth order and by two mechanical constrains related with the variation of rotational speeds at the DFIG shaft, $\omega_{m G}$ and at the IM shaft, $\omega_{m M}$. Its controllability has been also verified and simulation results and experimental validation has been carried out in early works [3].

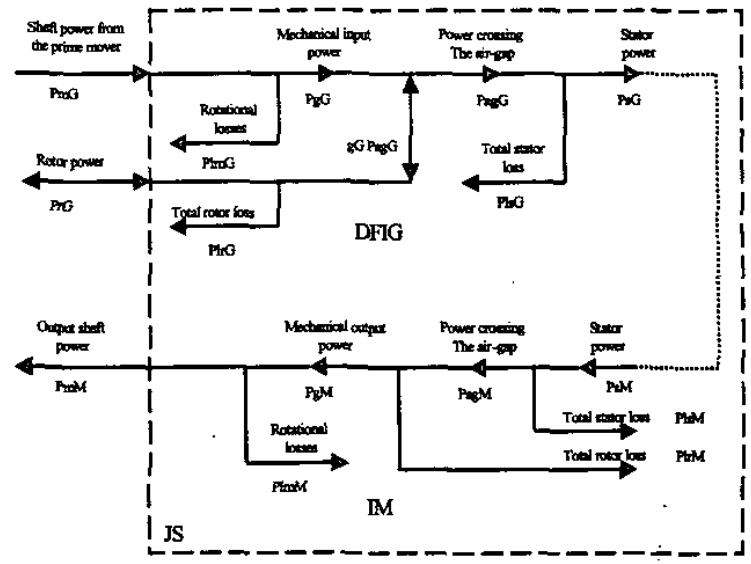

Figure 3. Power flow diagram for the JS

\section{- IV. POWER FLOW IN THE JS}

The main objective of the JS shown in Figure 2, is to generate mechanical speed, $\omega_{m M}$ and torque $\Gamma_{m M}$, using its hybrid energy resources (mechanical from the prime mover and electrical from the battery) in the best way.

The operation mode chosen for the JS to work depends on the speed level demand, $\omega_{m M}$ deman, for the load connected to the motor shaft. In this way, it can be established two operation modes: the normal mode, and the battery mode.

\section{Normal mode}

The DFIG is switched on, the prime mover speed $\omega_{m G}$ is held constant; and the power drawn from the converter at the rotor side, named slip power, is derived from the batteries. This operation mode includes two operation submodes:

Subsynchronous mode. The DFIG is working at subsynchronous speed, so generator slip has positive value, $g G>0$. The power transmitted to the air-gap, PagG, is greater than the mechanical input power obtained from the prime mover. This is because the secondary source is providing a slip power, $g G . P a g G$, from the batteries. The maximum power available at the output is the sum of $P a g G$ and $g G . P a g G,(1+g G) . P a g G$, minus the losses in each step of energy conversion. The maximum speed available at the output is $\omega_{m M M}$. The minimum speed available at the output system in this mode, $\omega_{m M}$ SINC, $_{\text {, }}$ is obtained when the DFIG is running near synchronism.

Supersynchronous mode. The DFIG is working at supersynchronous speed, so $g G<0$ is verified. The power crossing the air-gap, $P a g G$, is less than the mechanical input power obtained from the prime mover. The excess power gG.PagG is returned to the secondary circuit to provide recharging power to the batteries. The maximum speed available at the output is $\omega_{m M}$ SFNC and the minimum $\omega_{m M M N}$ is obtained near $g G=-1$. 


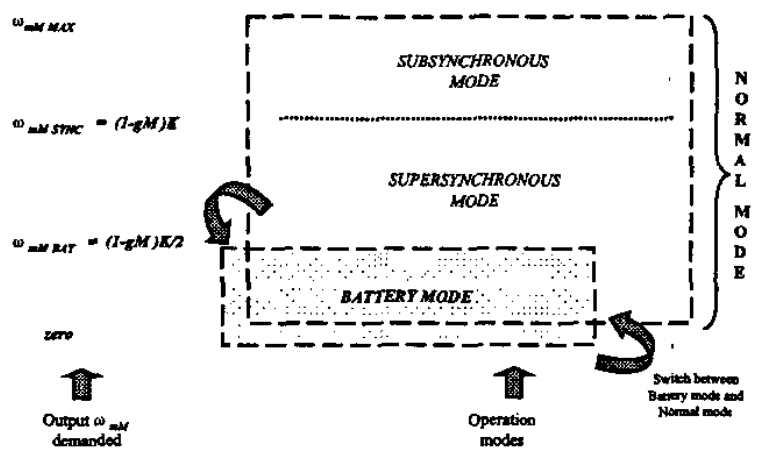

Figure 4. Speed range operation diagram for the JS depending on $K=$ constant speed of the ICE.

\section{Battery mode}

The DFIG is at standstill, because the prime mover speed $\omega_{m G}$ is zero, so $g G=1$, thereby DFIG is behaving like a frequency-changer system.

The power crossing the air-gap is only the power delivered by the batteries. Obviously, the maximum power available at the output is the battery power minus the losses in each step of energy conversion.

In Figure 3 power flow in the JS is illustrated for both operation modes (normal mode and battery mode). Double arrow for the generator rotor power $\operatorname{PrG}$, indicates the dual possibility to provide or return power from/to the battery in subsynchronous mode and supersynchronous mode respectively.

Now, it is possible to determine the limits of operation of the JS as a function of the demanded speed $\omega_{m} G$ deman .

Considering that the $\mathrm{IM}$ is nunning in subsynchronous mode, $0<g M<1$ is verified and

$$
\omega_{m M}=(1-g M) \omega_{s M}=(1-g M)\left(\omega_{r G}+\omega_{m G}\right)
$$

On the other hand, $\omega_{r G}=g G \omega_{s G}$ is the input rotor speed, and $\omega_{m G}=K=$ constant is the mechanical speed of the prime mover held constant to optimize its performance. Finally,

$\omega_{m M}=\frac{(1-g M) K}{(1-g G)}$

For the upper limit of operation, in the subsynchronous mode, gG $\rightarrow 1$, that implies that $\omega_{m M} \rightarrow \infty$, which is not possible, so as far as speed system limit is concerned, the upper limit is determined by its power limit. This power limit is set by the combination of the voltage and current ratings with the power factors and efficiencies of the machines.

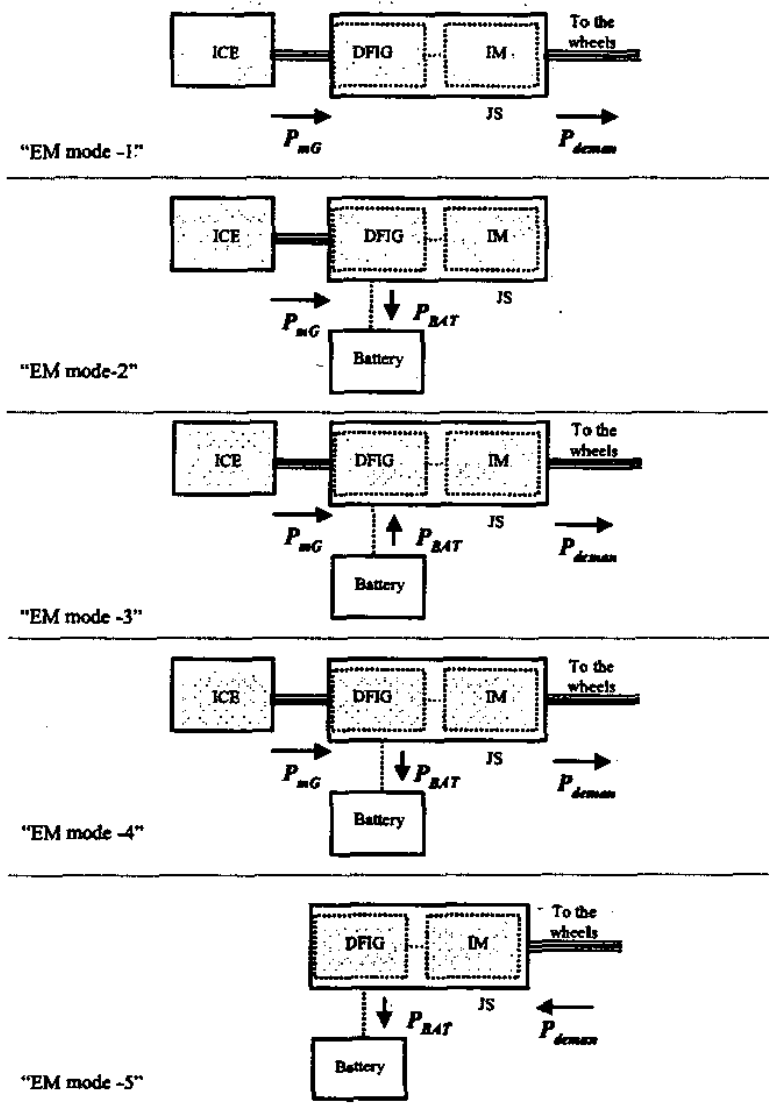

Figure 5. Power flow for all the five basic "EM modes"

$\omega_{m M M X}=\max \left[\omega_{m M}\right]$

For the synchronous point, when system is crossing from subsynchronous mode to supersynchronous mode, the condition $g G=0$ is verified, so considering (2) yields

$\omega_{m M S Y N C}=(1-g M) K$

For the lower limit of normal mode, below which the system is running in battery mode, the condition $g G=-I$ is verified, so considering ( 1 ) yields

$\omega_{m M S Y N C}=(1-g M) K / 2$

Forcing JS to run in specific modes of operation allows that hybrid power capacity can be better used. A fully defined speed range of operation is presented in Figure 4. 

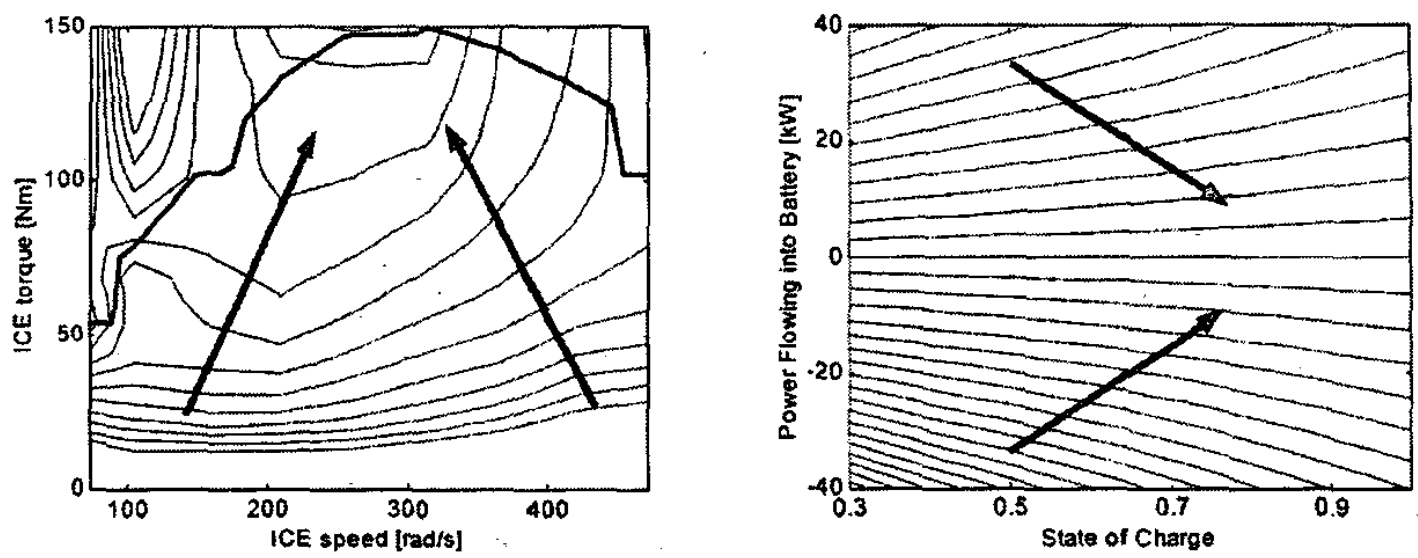

Figure 6: Efficiency Maps and Optimal Curves of a) the ICE, and b) the BAT. The arrows indicate increasing efficiency.

\section{ENERGY MANAGEMENT STRATEGY}

The basic idea of a hybrid vehicle is to decouple the energy source (in the ICE and the BAT) from the energy utilization (in the wheels) by an intermediate stage (the JS). The energy contained in the fuel is transformed to mechanical energy (ICE) and by a generating element (the DFIG) in electric energy, and the ultimate conversion process from electric to mechanic energy is performed independently (in the IM). A BAT delivers energy only during the power peaks. The advantages of this generation scheme rely on the reduced size of the batteries and on the constant speed working conditions of the ICE that ensures low gas emission and efficient consumption of fuel.

The rated power of the generating unit in the HEV is predetermined to the estimated required mean power in order to ideally complete a working cycle maintaining the state of charge (SOC) of the battery at a certain level [4].

Figure 1 also shows that the series hybrid drive train includes an Energy Management Controller, EMC. The EMC in the rotor of the DFIG is becoming the key element for distributing and controlling the electrical energy in energy subsystems, including the battery, for the HEV environment, with the extra advantage that it can be implemented digitally on a low cost processor.

The main objective of the EMC is to satisfy the required power from the propulsion system while keeping fuel consumption and vehicle emissions as.low as possible.

The presence of two energy sources and the possibility to recover energy during the braking permits at least five different ways to operate the propulsion system, depending on the flow of energy. Figure 5 shows these five basic modes for a Series HEV with JS to work. Three modes (EM modes 1,3 and 4) correspond to constant march and accelerations of the vehicle, and two modes (EM modes 2 and 5) correspond to the vehicle at stall or braking.

Under the Ruled-Based Power Split strategy the power source will be assigned to the ICE, the BAT, or to a combination of both, based not only on the SOC status, but also on the Power Demand from wheels, Pdeman.

The Pdeman from wheels is positive during acceleration and cruise phases of the drive cycle and is negative during the deceleration phase of the drive cycle.

The EMC should determine how much power is need to drive the wheels based on the Acceleration Command, and how much is need to charge the battery, based on the SOC. Then it should split the power between ICE and Bat. If the battery needs to be charged, the ICE should provide the power to both driving the wheels and charging the battery.

The power-split strategy can be used to optimize the efficiency of all three basic components of the HEV (ICE, BAT and IM), because it determines the operating points of the components. To identify the optimal points, efficiency maps of the components should be obtained previously.

Figure 6 shows efficient maps taken from [5]. Once the efficiency characteristics of the components are known, it is possible to formulate the power-split strategy.

\section{ENERGY MANAGEMENT CONTROLLER}

The general energy management strategy should be implemented in a EMC using some kind of heuristic logic or rule-based approach. Authors propose an EMC which contains a control law that is described by a knowledge-based system consisting of "If ... Then" rules with vague predicates and a logic inference mechanism, so, the rule-base is the main part of the EMC. 


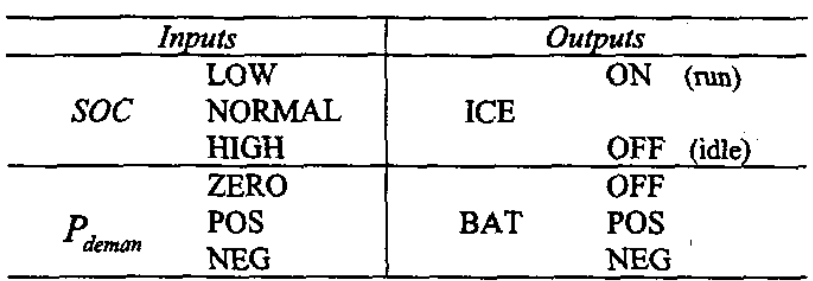

Table 1: Linguistic labels for input and output variables.

It is formed by a family of logical rules that describes the relationship between the inputs ( $S O C$ and $P$ deman), and the outputs (ICE status, BAT status) of the controller.

Possible linguistic labels that form the term set of each input and output variable is presented in Table 1.

Pdeman is "ZERO" when the vehicle is stall, "POS" when it is running at any speed, and "NEG" when it is braking.

The levels for the $S O C$ mean "LOW" when the battery reaches the minimum level of charge, "NORMAL" when it is between limits, and "HIGH" when it reaches the maximum level of charge. The levels for the BAT mean "OFF" when disconnected, "POS" when it is charging and "NEG" when it is discharging.

Based on the status of the inputs, the assignment of power is determined as follows from Table 2 , where only some interesting status of the system have been represented.

For more complicated control issues it is need a more detailed rule-base, i.e. a more complete description of the control strategy.

\section{If $\mathrm{SOC}=$ "NORMAL" and $P_{\text {deman }}=$ "POS" then ICE is "ON" and BAT is "OFF"}
2 If $\mathrm{SOC}=$ "LOW" and $P_{\text {demam }}=$ "ZERO" then ICE is "ON" and BAT is "POS"
3 If $\mathrm{SOC}=$ "HIGH" and $P_{\text {denam }}=$ "POS" then ICE is "ON" and BAT is "NEG"

4 If $\mathrm{SOC}=$ "LOW" and $P_{\text {demam }}=$ "POS" then ICE is "ON" and BAT is "POS"

5 If $\mathrm{SOC}=$ "LOW" and $P_{\text {deman }}=$ "NEG" then ICE is "OFF" and BAT is "POS"

Table 2: Set of the utilized control rules for the EMC

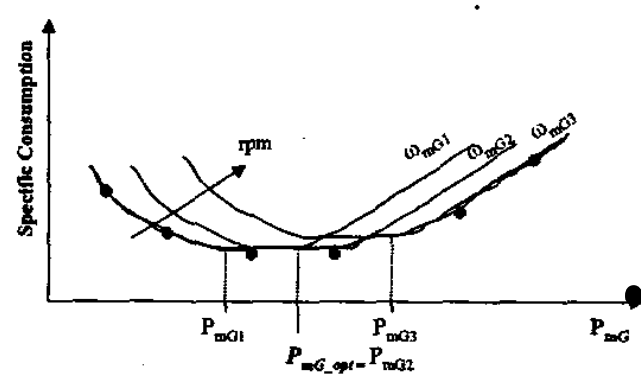

- Variable speed, minimum consumption of the ICE

Figure 7: Qualitative specific consumption of the ICE

The ICE specific consumption, considering different rotational speeds, $\omega_{m G}$ has a qualitative behavior of the type depicted in Figure 7 [6].

If for each ICE power, $P m G$, the optimal speed is considered, the variable speed specific consumption curve becomes the envelope, lower curve shown in figure (indicated with black crosses).

The control strategy consists, therefore, in operate the ICE so that it follows the minimum consumption curve by varying its rotational speed in predetermined values of $\omega_{m G}$ when following the required power Pdeman. The determination of the better values $\omega_{m G 1}, \omega_{m G 2}$ and $\omega_{m G 3}$ must be done in function of the optimal range of running for the ICE.

The optimum power of the ICE to obtain minimum fuel consumption is $P m G$ opt, that is the minimum of the envelope curve, that corresponds in this case to the minimum of the curve, $\omega_{m G 2}$ so $P m G$ opt $=P m G 2$ is verified.

The controller should act differently in the cases that drive power request by the load, Pdeman, is lower or greater than the optimum power identified by the point $P m G$ opt . The EMC rule-base from Table 2 can be significant improved if these considerations are taken into account.

All the rules of Table 2 can be replaced by a more particularized description formed by more than one rule. The increased granularity of the term set of the input and output variable results in a finer formulated logical statements.

A new term set with different linguistic labels for input $P$ deman and output $I C E$ is presented in Table 3, where the meaning of the different levels for $P$ deman can be explain as follows:

- level "VERY LOW" is presented when $P$ deman $<P m G l$,

- level "LOW" is presented when $P m G I<P d e m a n$ $<P m G$ opt,

- level "NORMAL" is presented when $P$ deman $=P m G$ opt,

- level "HIGH" is presented when $P m G \_o p t<$ $P$ deman $<P m G 3$,

- level "VERY HIGH" is presented when $P m G 3<P$ deman. 


\begin{tabular}{|c|c|c|c|}
\hline \multicolumn{2}{|r|}{ Inputs } & \multicolumn{2}{|c|}{ Outputs } \\
\hline$S O C$ & $\begin{array}{l}\text { LOW } \\
\text { NORMAL } \\
\text { HIGH }\end{array}$ & ICE & $\begin{array}{l}\text { LOW } \\
\text { OPT } \\
\text { HIGH } \\
\text { OFF }\end{array}$ \\
\hline$P_{\text {deman }}$ & $\begin{array}{l}\text { ZERO } \\
\text { VERY LOW } \\
\text { LOW } \\
\text { NORMAL } \\
\text { HIGH } \\
\text { VERY HIGH } \\
\text { NEG }\end{array}$ & BAT & $\begin{array}{l}\text { OFF } \\
\text { POS } \\
\text { NEG }\end{array}$ \\
\hline
\end{tabular}

The levels for the ICE mean: "LOW" when $I C E=P m G 1$, "OPT" when $I C E=P m G o p t$, and "HIGH" when $I C E=P m G 3$.

Tables 4 represents an improved description of the rules of Table 2.

\section{SIMULATION OF A SERIES HEV WITH JS IN THE DRIVETRAIN}

A search of the recent literature reveals that a number of computer software simulators are available specifically for HEVs. One of the more prominent tools is ADVISOR, developed by the NREL in 1994. This program is flexible enough to operate in the popular MATLAB/SIMULINK programming enviroment and is used in this work to simulate the behavior of the JS in a Series HEV model. Figure 8 shows the Data Flow Scheme used in the simulation of a Series HEV with the inclusion of the JS in the drivetrain and Figure 9 shows the basic ADVISOR scheme implemented for the purpose of validate the power flow behavior of the JS

The theoretical vehicle used in this work to test the behavior of the JS is a default Series HEV. The vehicle typically operates with an on-board generation system formed by the ICE and the DFIG, which maintains the batteries at an acceptable SOC.

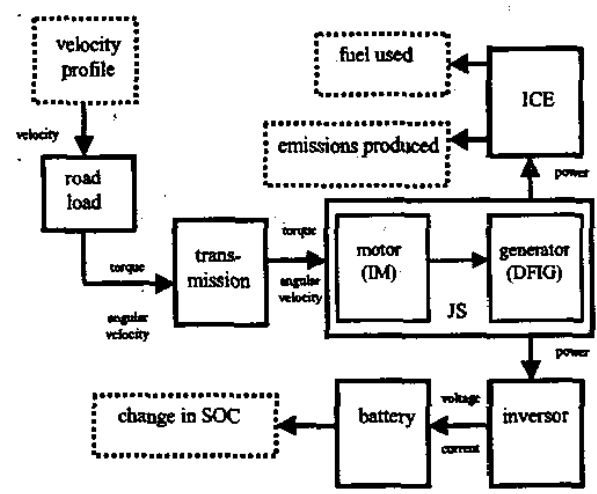

Figure 8. ADVISOR Data Flow for a Series HEV with JS
1-1 If the "HIGH" SOC value is reached and Pdeman is "VERY LOW"

.......then ICE is "LOW" $(P I C E==P m G 1)$

........ and BAT is "OFF"(Ploaddump $=P m G 1$-Pdeman $)$

1-2 If the "HIGH" SOC value is reached and $P$ deman is "LOW"

........ then ICE is "LOW" $(P I C E==P m G I)$ ........ and BAT is "NEG" (Pbatt $=P m$ G1-Pdeman)

2-1 If the "LOW" SOC value is reached and Pdeman is "VERY LOW"

............ then ICE is "OPT" (PICE=PmGopt) ............ and BAT is "POS" (Pbatt=PmGopt-Pdeman)

2-2 If the "LOW" SOC value is reached and Pdeman is "LOW" then ICE is "OPT" (PICE=PmGopt) and BAT is "POS" (Pbatt=PmGopt-Pdeman) (Note that in this case ICE should to run at "LOW" but is running at "OPT" to prevent the possibility of SOC $=$ "LOW" and Pdeman= "VERY HIGH")

2-3 If the "NORMAL" SOC value is reached and Pdeman is "VERY LOW"

............. then ICE is "LOW" (PICE $=$ PmG1)

............. and BAT is "POS" (Pbatt=PmG1-Pdeman)

2-4 If the "NORMAL" SOC value is reached and Pdeman is "LOW"

............ then ICE is "OPT" (PICE=PmGopt)

............ and BAT is "POS" (Pbatt-PmGopt-Pdeman)

3-1 If the "NORMAL" SOC value is reached and Pdeman is "HIGH" then ICE is "OPT" (PICE $=$ PmGopt) ........... and BAT is "NEG" (Pbatt=PmGopt-Pdeman)

3-2 If the "NORMAL" SOC value is reached and Pdeman is "VERY HIGH"

............ then ICE is " $\mathrm{HIGH}$ " (PICE $=\mathrm{PmG} 3$ )

.............. and BAT is "NEG" (Pbatt=PmG3-Pdeman)

3-3 If the "HIGH" SOC value is reached and Pdeman is "HIGH"

........... then ICE is "OPT" (PICE=PmGopt) ........... and BAT is "NEG" (Pbatt=PmGopt-Pdeman)

3-4 If the "HIGH" SOC value is reached and Pdeman is "VERY HIGH" ........... then ICE is "OPT" (PICE=PmGopt) ............ and BAT is "NEG" (Pbatt=PmGopt-Pdeman) (Note that in this case ICE should to nun at "HIGH" but is running at "OPT" taking advantage to the fact that SOC is "HIGH", to improve fuel consumption and to reduce harmful emissions.)

4-1 If the "LOW" SOC value is reached and Pdeman is "HIGH"

............ then ICE is "HIGH" (PICE=PmG3) .............. and BAT is ' $\mathrm{POS}$ " (Pbatt=PmG3-Pdeman)

4-2 If the "LOW" SOC value is reached and Pdeman is "VERY HIGH"

(This possibility is avoided by the extra-charging of the battery in the case 2-2)

Table 4. Set of the of improved control rules for the EMC: 


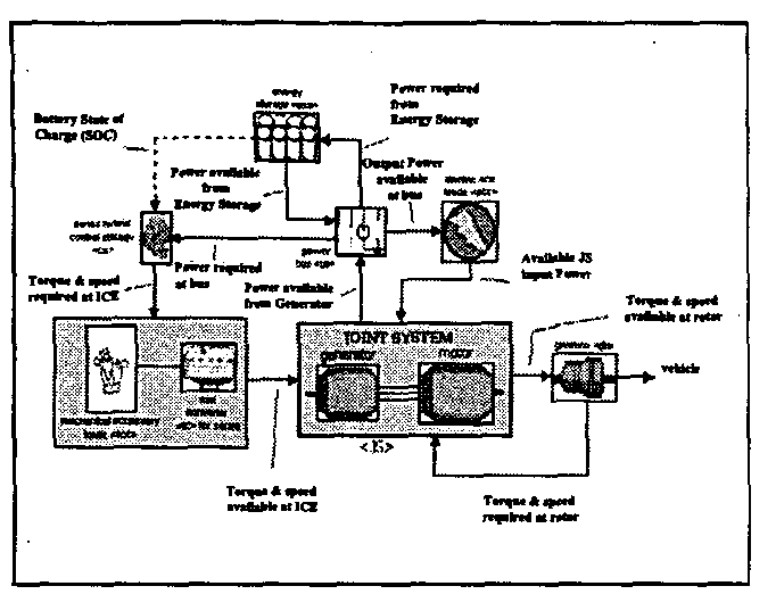

Figure 9. ADVISOR scheme of a Series HEV with JS

Under normal operation, when the ICE/DFIG controller senses a low battery SOC, it executes a start-up procedure for the engine, then maintains the engine speed at the proper level to produce power until the batteries reach a high SOC. It then shuts down the engine and continues to monitor battery SOC. Next, a brief description of some of the modeled subsystems of the HEV is presented.

Joint System. The JS block translates torque and speed request into electric power requests and convert actual power input to torque and speed output.

RC Battery Model. The Energy Storage System (ESS) block represents the battery pack that stores energy on-board the modeled vehicle. This block accepts the power request and returns available power output from the battery and battery SOC. By convention, positive power is discharge. The RC model uses power as an input and limits the battery operation to the high and low voltage limits.

Series Hybrid Control Strategy. The energy management control strategy described in Section VI uses the JS and the fuel converter to generate electrical energy for use by the vehicle. The SOC is input into the block, and the required ICE torque and speed are the outputs.

Simulation results. A CYC_TEST_10sec driving cycle is used to test the JS performance in a HEV with the ADVISOR platform. Figure 10 shows this driving cycle required to the HEV and also shows the effective speed achieved by the vehicle when powered by the JS.

Note the small discrepancy between both curves at the maximum demanded speed. This difference is due to the small rated IM.

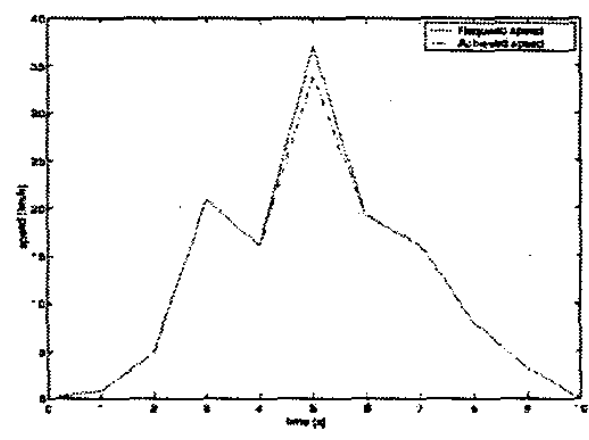

Figura10. Dynamic performance of the Series HEV with JS

Battery charging and discharging. In order to verify the charging and discharging of the battery, a set of simulations have been carried out with MATLAB/SIMULINK. Table 5 shows the results obtained working at different modes of the JS. All the tests show the possibility of switch between charge (positive sign of P BAT) and discharge (negative sign of P BAT) of the battery. In test $\# 1$, variations in the mechanical speed of the $\mathrm{IM}, \omega_{m M}$, are considered. In test $\# 2$, variations in the rotor speed of the DFIG, $\omega_{r G}$, are considered. In test \#3, two mechanical ICE speeds $\omega_{m G l}$ and $\omega_{m G 2}$ are considered. Finally, test \#4 shows a possible variation in the climbing coefficient $K O$ of the road, where the sign is positive when the vehicle is climbing up and the sign is negative when the vehicle is down, in regenerating braking.

In the last column is also indicated the power demanded at the wheels, Pdeman.

Results obtained validated the energy management strategies developed.

\begin{tabular}{|c|c|c|c|c|c|c|}
\hline $\begin{array}{c}\text { Test } \\
\#\end{array}$ & $\omega_{\mathrm{mm}}$ & $\begin{array}{c}\omega_{\mathrm{rO}} \\
{[\mathrm{rad} / \mathrm{secl}}\end{array}$ & $\omega_{\operatorname{ming}}$ & K0 & Pntw & $\begin{array}{c}\text { P deman } \\
{[\text { [w] }}\end{array}$ \\
\hline 1 & $\begin{array}{l}76.0 \\
78.0\end{array}$ & $\begin{array}{l}-157.1 \\
-157.1\end{array}$ & $\begin{array}{l}250.0 \\
250.0\end{array}$ & $\begin{array}{l}0.2 \\
0.2\end{array}$ & 7.7. & $\begin{array}{l}44.0 \\
46.6\end{array}$ \\
\hline 2 & $\begin{array}{l}58.0 \\
89.0\end{array}$ & $\begin{array}{l}-144.5 \\
-125.6\end{array}$ & $\begin{array}{l}250.0 \\
250.0\end{array}$ & $\begin{array}{l}0.2 \\
0.2\end{array}$ & 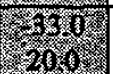 & $\begin{array}{l}28.6 \\
58.0\end{array}$ \\
\hline 3 & $\begin{array}{l}75.0 \\
76.0\end{array}$ & $\begin{array}{l}-157.1 \\
-157.1\end{array}$ & $\begin{array}{l}320.0 \\
330.0\end{array}$ & $\begin{array}{l}0.2 \\
0.2\end{array}$ & 6. & $\begin{array}{l}43.0 \\
44.6\end{array}$ \\
\hline 4 & $\begin{array}{l}68.0 \\
83.0\end{array}$ & $\begin{array}{l}-157.1 \\
-157.1\end{array}$ & $\begin{array}{l}250.0 \\
250.0\end{array}$ & $\begin{array}{r}0.0 \\
-0.2\end{array}$ & 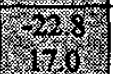 & $\begin{array}{c}23.4 \\
-10.0\end{array}$ \\
\hline
\end{tabular}

Table 5. Battery power variations in the different operation modes 


\section{CONCLUSIONS}

The main objective of this work is to show the use of the specially designed motion system JS in a specific propulsion configuration, such as HEVs, where JS has demonstrated to meet the specific performance requirements.

The work developed in this paper shows also the suitability of rule-based control techniques for the energy management of an HEV. The use of intelligent control is justified because HEV drivetrains represent highly nonlinear multidomain systems. The power-split concept allows to employ a control strategy to both ICE and JS. The rule-base set can be significant improved if the optimum power of the ICE is taken into account to obtain minimum fuel consumption.

ADVISOR simulations of the JS working in a Series HEV have been carried out to analyze energy consumption. Modifications have been made to the ADVISOR code to allow to the use of the JS model. Basically the feed-forward model of the JS has been modified to allow its linking with the vehicle model and to integrate vehicle and JS simulations. The obtained results have been very promising.

\section{ACKNOWLEDGMENTS}

This work was partially support by Bilateral Cooperation Program Conacyt-Csic E130.866/2002 and by CICYT DPI2000-1509-C03-02.

\section{REFERENCES}

[1] Chan, C. "The state of the art of electric and hybrid vehicles", in Proceedings of the IEEE, Vol.90, No.2, pp. 247-275, February 2002.

[2] Tnani, S., S. Diop, S. Jones, A. Berthon . "Novel Control Strategy of Double - Fed Induction Machines", Proc. of EPE'95, Sevilla, Spain, pp.1553-1558. 1995

[3] Caratozzolo, P., Fossas, E., Pedra, J., Riera , J. "Dynamic Modeling of an Isolated Motion System with DFIG', Proceedings of the CIEP'OO, Acapulco, México, pp. 287--251. 1995.

[4] Cernuto, E., Consoli, A., Raciti, A., Testa, A. "Fuzzy logic based efficiency improvement of an urban electric vehicle" Proceedings of 20th International Conference on the Industrial Electronics, Control and Instrumentation, IECON '94., vol.2, pp. 1304-1309, 1994.

[5] Schouten,N., Salman, M., Kheir, N. "Fuzzy Logic Control for Parallel Hybrid Vehicles", Trans. On Control Systems Technology, Vol. 10, No.3, pp. 460-468, May 2002

[6] Barsali, S., Ceraolo, M., Possenti, A. "Techniques to control electricity Generation in a Series Hybrid Electrical Vehicle", Trans. on Energy" Conversion, Vol.17, No.2, pp. 260-266, June 2002. 